\section{Comparative evaluation of biological human leukocyte antigen DPB1 mismatch models for sur- vival and graft-versus-host disease prediction after unrelated donor hematopoietic cell transplantation}

Hematopoietic cell transplantation (HCT) from unrelated donors (UD) is a curative therapy for many hematologic malignancies. Donor-recipient allele-level matching at human leukocyte antigen (HLA)-A, -B, -C, -DRB1 $(8 / 8)$ is widely accepted to provide best results in terms of overall survival (OS), non-relapse mortality (NRM) and graft-versus-host disease (GvHD). ${ }^{1}$ Genotypic matching for HLA-DPB1 (DPB1) has been shown to hold limited, if any, impact on post-transplant OS, and would be challenging to adopt, due to the weak linkage disequilibrium between DPB1 and the remaining HLA class II loci. Therefore, more than $80 \%$ of $8 / 8$-matched UD-HCT are currently DPB1-mismatched. ${ }^{1}$ A number of studies have demonstrated that biological models can be used to identify selected, permissive DPB1 mismatch combinations, associated with lower clinical risks compared to their high-risk, non-permissive, counterparts. ${ }^{2-9}$ Five different biological models for the assignment of DPB1 permissiveness have been identified to date, three of which are based on functional T-cell epitopes (TCE), while the remaining two rely on a single nucleotide polymorphism (SNP) tag for expression levels. In particular, in the TCE3 model, DPB1 alleles are classified into three structural groups based on variation in the peptide antigen-binding domain, which leads to either functionally similar or distinct behavior in terms of T-cell alloreactivity. ${ }^{2-5}$ The TCE4 model is identical to the TCE3 model, except for the assignment of $\mathrm{DPB} 1{ }^{*} 02$ to a fourth, independent group 4. Another derivative of TCE3 is the "delta functional distance" $(\triangle \mathrm{FD})$ model, in which a functional distance (FD) score is assigned to every DPB1 allele based on key polymorphic aminoacids involved in anti-DPB1 alloreactivity, and differences between the FD of patient and donor alleles defines permissive and non-permissive pairs, respectively., ${ }^{6,7}$ The SNP models, in contrast, are based on high or low expression DPB1 alleles according to a SNP tag in the 3' untranslated region, in which the rs9277534 A and G variant is associated with low and high DPB1 expression, respectively. ${ }^{8-9}$ In the expression model, this SNP tag is applied to all DPB1 alleles, while in the DP2/DP5 model, it is applied to 19 DPB1 alleles belonging either to the DP2 or the DP5 evolutionary clade. Currently, TCE3 matching is proposed by stem cell donor registries in the USA and Europe, because it is the only one of these models which has been validated in independent cohorts. However, a comparative evaluation of the five different biological models for DPB1 permissiveness and their association with HCT outcome has not been undertaken so far.

Here, we have filled this gap by analysing the outcome of 422 patients with available second field DPB1 typing transplanted from 8/8-matched HLA-A, -B, -C and DRB1 allele matched UD in 32 centers from the Gruppo Italiano Trapianto di Midollo Osseo (GITMO), between 2012 and 2015. Of these, 43 pairs had a mismatch at HLA-DOB1, and 382 had one or two DPB1 mismatches. The availability of DPB1 typing did not introduce significant biases, since clinical outcomes were similar for the 422 transplants under analysis and those $(n=522)$ performed in the same time-period and for which DPB1 typing was not available (Online Supplementary Table S1A-B). Patient, donor and transplant characteristics are shown in Table 1. Transplants were performed for hematologic malignancies, with mostly myeloablative conditioning and peripheral blood as stem cell source. GvHD prophylaxis included anti-T-lymphocytic globulin (ATG) in most cases. Permissive and non-permissive mismatches were assigned by the IMGT webtool version 2.0 for TCE3, and manually for the other models, using the cut-off 1.64 or 2.665 for $\Delta \mathrm{FD}$ as described; ${ }^{6,7}$ for the Expression model, the rs9277534 SNP variant was predicted by DPB1 genotyping. ${ }^{8}$ Statistical methods are described in the Online Supplementary Materials and Methods.

Table 1. Patient, transplant and donor characteristics.

Population $n=422$

\begin{tabular}{lc} 
Median follow-up for survivors, years (range) & $3.2(0.1-6)$ \\
Patient age, years, median (range) & $49(18-70)$ \\
\hline Patient sex, male, n (\%) & $244(58 \%)$ \\
Type of diagnosis, n (\%) & \\
AML & $168(40 \%)$ \\
ALL & $63(15 \%)$ \\
MDS or MPN & $69(16 \%)$ \\
Lymphoma and Myeloma & $110(26 \%)$ \\
CLL & $12(3 \%)$ \\
\hline
\end{tabular}

Disease status according to EBMT risk ${ }^{14}, \mathrm{n}(\%)$

Early

$191(45 \%)$

Intermediate

$111(26 \%)$

Advanced

HCT-CI score ${ }^{15}$, median (range) $1(0-7)$

Karnofsky performance status, median (range) $90 \%$ (50-100)

Donor sex, male, $n(\%) \quad 306$ (72\%)

$\mathrm{N}^{\circ}$ of previous pregnancies $\quad 0(0-6$

for female donors, median (range)

Female donor/male recipient, $\mathrm{n}(\%)$

$61(14 \%)$

Host/donor CMV serostatus, n(\%)

Pos/pos

$157(37 \%)$

Pos/neg

$166(39 \%)$

Neg/pos

Neg/neg

$53(13 \%)$

Missing

Type of conditioning, $\mathrm{n}(\%)$

MAC

$271(64 \%)$

RIC

$111(35 \%)$

Source of stem cells, n (\%)

PB $343(81 \%)$

$\mathrm{BM}$

$79(19 \%)$

ATG-based GvHD prophylaxis, $\mathrm{n}(\%) \quad 382(91 \%)$

GvHD prophylaxis details

$\mathrm{ATG}+\mathrm{CSA}+\mathrm{MTX}$

$341(81 \%)$

ATG+Sirolimus+MMF

Other ATG-based prophylaxis

CSA+MTX

Other prophylaxis

$16(4 \%)$

AML: acute myeloid leukemia; ALL: acute lymphoblastic leukemia; MDS: myelodysplastic syndromes; MPN: myeloproliferative neoplasms; CLL: chronic lymphocytic leukemia; CMV: cytomegalovirus; MAC: myeloablative conditioning; RIC: reduced intensity conditioning; PB: peripheral blood; BM: bone marrow; ATG: anti-T-lymphocytic globulin; GvHD: graft-versus-host disease; CSA: cyclosporine A; MTX Methotrexate; MMF: Mycophenolate Mofetil. 
The five models displayed a limited overlap, and the TCE4 model was the most restrictive one, as in this model the lowest number of pairs $(36 \%)$ were classified as permissive. For the SNP models, only donor-recipient pairs with a single unidirectional DPB1 mismatch in GvH direction could be classified, leaving 153 of 382 (40\%) and 233 of $382(61 \%)$ pairs without classification according to either the Expression model or the DP2/DP5 model, respectively (Figure 1A).

Donor-recipient pairs in the permissive/low risk or non-permissive/high risk groups according to all five models were comparable with regards to disease- and

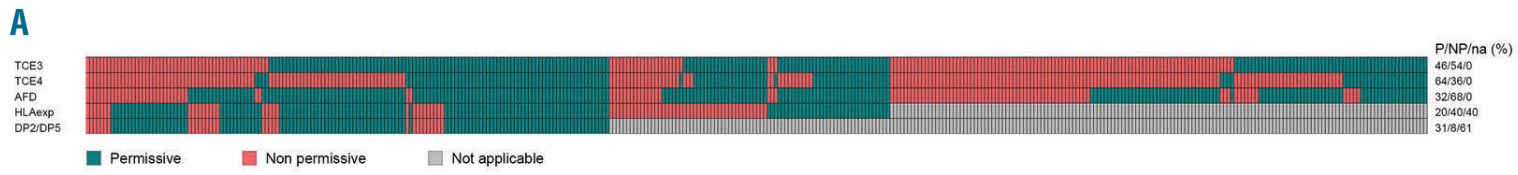

B Overall survival

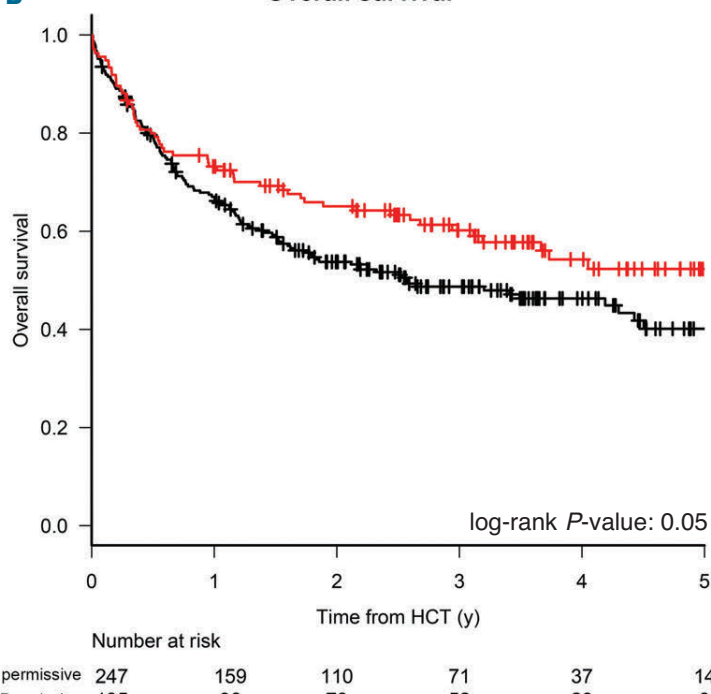

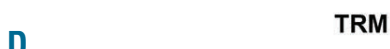

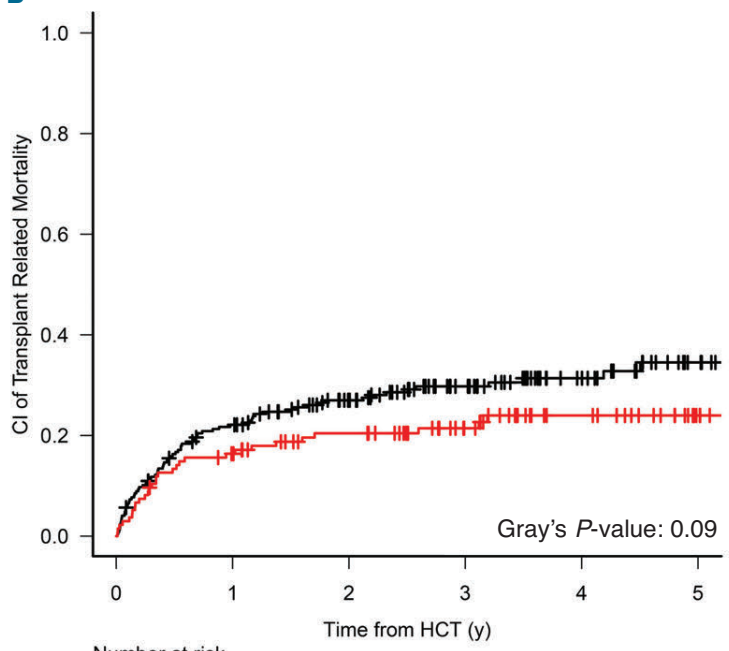

Number at risk
TRM

95
29
C

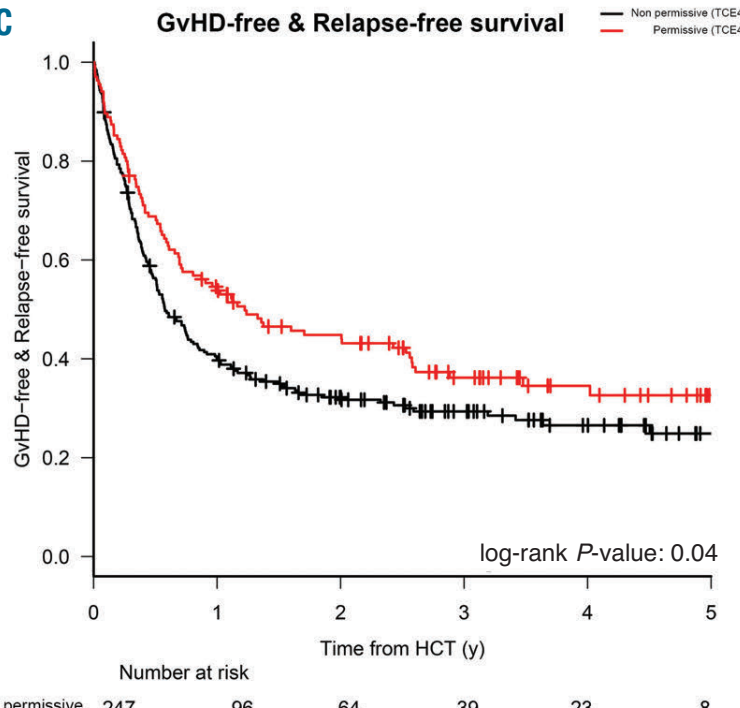

GvHD-free \& Relapse-free survival 二

\section{$\mathbf{E}$}

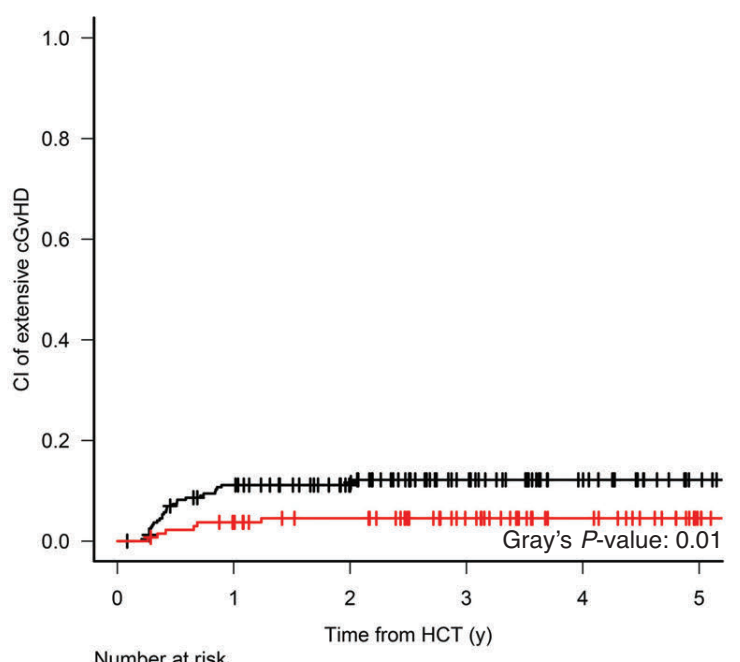

Number at risk

Non permissive 247 Permissive 135

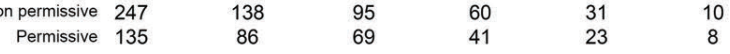

Figure 1. Comparative stratification of donor-recipient pairs according to five different biological models of DPB1 permissiveness, and outcome associations for TCE4. (A) Classification of the 382 DPB1 mismatched pairs under analysis in this study, as permissive or low risk (green) or as non-permissive or high risk (red) group according to five different biological models of permissiveness, as described in the text. For the Expression model and the DP2/DP5 model, only pairs with single mismatches in the graft-versus-host (GvH) direction can be stratified, the others cannot be classified (grey). The model under investigation, and the relevant numbers in each category (green, red or grey) are indicated to the left and to the right of the panel, respectively. The bottom panels show KaplanMeier probabilities for overall survival (OS) (B), graft-versus-host disease-free relapse-free survival (GRFS) (C), and commorbidity index (CI) of non-relapse mortality (NRM) (D) and extensive chronic graft-versus-host disease (cGvHD) (E) stratified for functional DPB1 matching according to TCE4 model, with permissive and non-permissive groups represented by the red and black curves, respectively.TRM: transplant-related mortality; HCT: hematopoietic cell transplantation; y: years. 
Table 2. Multivariate analysis of DPB1 mismatch models and association with hematopoietic cell transplantation outcomes.

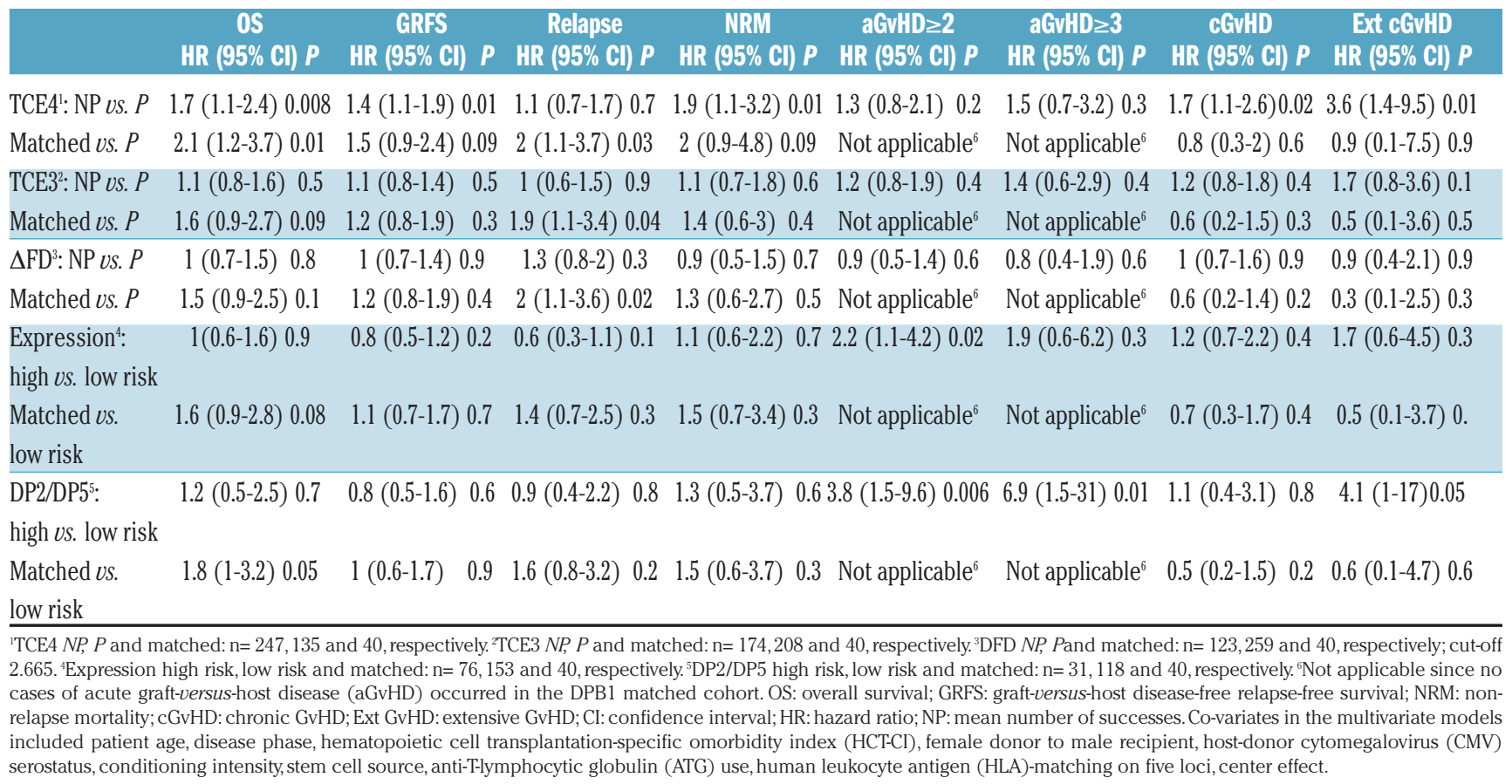

transplant-specific characteristics (Online Supplementary Tables S2-6). In univariate analysis, we confirmed previous reports ${ }^{10}$ that DPB1 allele mismatches were not associated with any significant difference in OS, and this was reflected by a balance between significantly higher risks of acute GvHD (aGvHD), in the presence of a markedly though not significantly lower risks of relapse (Online Supplementary Table S7). Of all models, only the TCE4 model was significantly associated with superior 3-year OS and GvHD-free relapse-free survival (GRFS) in patients transplanted from a permissive compared with a non-permissive donor, reflected by lower 3-year cumulative incidence $(\mathrm{CI})$ of extensive chronic GvHD (cGvHD) and NRM (Figure 1B-E). No significant associations with clinical outcomes were found for the TCE3 or the $\triangle F D$ model (Online Supplementary Table S7). The Expression model and the DP2/DP5 model were both associated with a higher 100-day CI of grade $\geq 2 \mathrm{aGvHD}$, but not with any of the other clinical endpoints (Online Supplementary Table S7). In multivariate analysis, TCE4 permissive pairs were independently associated with superior OS and GRFS, and with lower hazards for NRM, cGvHD and extensive cGvHD. Moreover, compared to DPB1 allele matches, permissive mismatches according to all three functional models (TCE3, TCE4 and $\triangle F D$ ) had significantly lower relapse risks (Table 2 ). In contrast, the high-risk mismatches according to the Expression model and the DP2/DP5 model were significantly associated with grade 2-4 aGvHD, but not with NRM or OS (Table 2). Outcome associations of all clinical co-variates used in the multivariate analyses are shown in the Online Supplementary Table S8.

Our study is the first to compare HCT outcome associations for the five major biological models of DPB1 permissiveness. The results show that the concordance on the predicted permissiveness of DPB1 mismatches among the different models is evident but far from outright, suggesting that, even if all models describe a com- mon biological phenomenon (the alloreactivity of $\mathrm{T}$ cells against incompatible DPB1 molecules), each of them may capture and emphasize only some aspects of this interaction. The outcome analyses confirm previous reports that functional DPB1 matching according to TCE is significantly associated with survival after UD-HCT2-5, while DPB1 matching according to the SNP tag predicts the risks of aGvHD. ${ }^{8-9}$ In this context. TCE4 is the most restrictive but appears as the best common denominator for permissiveness/low risk in all five models. In particular, the survival benefit of TCE4-permissive transplants over their non-permissive counterparts is mainly reflected by reduced NRM and cGvHD risks, providing support to the hypothesis that leveraging on permissive DPB1 mismatches might be a promising way to reduce NRM without compromising the graft-versus-malignancy effect of allogeneic HCT.

The study has several limitations. First, the number of pairs under analysis is relatively small, in particular for the two SNP tag models where DPB1 typing of up to $61 \%$ of pairs could not be classified. However, association of these two models with aGvHD risks is in agreement with previous reports from larger studies. ${ }^{8,9}$ Second, an apparent difference with previous data from larger studies $^{3,5}$ is the lack of association with TCE3. This might reflect the stem cell source, which was peripheral blood in $81 \%$ of our patients while bone marrow was used in at least $50 \%$ of patients from the other studies. ${ }^{3,5}$ Moreover, $91 \%$ of our patients received in vivo T-cell depletion with anti-thymocyte globulin (ATG), while this was adopted in less than $30 \%$ of patients in other studies. ${ }^{3,5}$ It should also be noted that TCE4 has been previously found to be associated with OS, including an analysis of non-overlapping Gruppo Italiano Trapianto Midollo Osseo (GITMO) transplants from earlier years, ${ }^{4}$ a recent study from the French Registry, ${ }^{11}$ and a large multicentre study from the International Histocompatibility Workshop. ${ }^{3}$ Since the latter did not 
show a significant advantage of TCE4 over TCE3, and DPB1 permissive donors are more frequent in TCE3, TCE3 was adopted by stem cell donor registries and not further investigated in subsequent studies. ${ }^{5}$ The greater restrictiveness of TCE4 compared to TCE3 in assigning permissiveness is due to appreciation of DPB $1 * 02$ as a separate functional group. Interestingly, HLA-DP2 is the so far only HLA-DP specificity associated with autoimmunity, ${ }^{12}$ and recent evidence suggests a similar breadth of the alloreactive T-cell receptor repertoire in permissive pairs involving this allele group compared to non-permissive pairs, ${ }^{13}$ arguing in favor of a functional basis for TCE4.

In conclusion, our results highlight the relevance of refining transplant-associated risks according to the biological significance of HLA matching. In particular, they confirm the association between the SNP tag models and aGvHD, while TCE4 should be prioritized for its highest performance in predicting survival and non-relapse related events. Moreover, since most of TCE4 permissive donors are classified as low-risk for both SNP tag models, they may be the best alternative to favor positive overall outcomes. Clearly, additional and possibly prospective studies should be performed to provide more definitive evidence for the respective value of the five DPB1 models, also in view of emerging new strategies for GvHD prophylaxis, which could modulate the observed outcome associations.

Francesca Lorentino, ${ }^{1}$ Nicoletta Sacchi, Elena Oldani, ${ }^{3}$ Valeria Miotti, ${ }^{4}$ Alessandra Picardi, ${ }^{5,6}$ Anna Maria Gallina, ${ }^{2}$ Pietro Crivello, ${ }^{7}$ Paolo Bernasconi, ${ }^{8}$ Riccardo Saccardi, ${ }^{9}$ Lucia Farina, ${ }^{10}$ Fabio Benedetti, ${ }^{11}$ Michela Cerno, ${ }^{4}$ Anna Grassi, ${ }^{3}$ Benedetto Bruno, ${ }^{12,13}$ Francesca Patriarca, ${ }^{4}$ Fabio Ciceri, ${ }^{1,14}$ Katharina Fleischhauer, ${ }^{7,1, \#}$ Luca Vago ${ }^{1,16, \#}$ and Francesca Bonifazi $i^{17, \#}$

"are co-senior authors

'Hematology and Bone Marrow Transplantation Unit, IRCCS San Raffaele Scientific Institute, Milano, Italy; 'Italian Bone Marrow Donor Registry, E.O. Galliera, Genova, Italy; ${ }^{3}$ Hematology and BMT Unit, Ospedale Papa Giovanni XXIII, Bergamo, Italy; ${ }^{4}$ Azienda Sanitaria Universitaria Integrata di Udine, Udine, Italy; ${ }^{5}$ Biomedicine and Prevention Department, Tor Vergata University, Roma, Italy; ${ }^{6}$ Hematology with Stem Cell Transplant Unit, AORN A. Cardarelli, Napoli, Italy; Institute for Experimental Cellular Therapy, Essen University Hospital, Essen, Germany; ${ }^{B}$ Bone Marrow Transplant Unit, Fondazione IRCCS Policlinico San Matteo, Pavia, Italy; ${ }^{\circ}$ Azienda Ospedaliero-Universitaria Careggi, Firenze, Italy; ${ }^{10}$ Hematology Department, Fondazione IRCCS Istituto Nazionale dei Tumori, Milano, Italy; "Department of Medicine, Section of Hematology and Bone Marrow Transplant Unit, University of Verona, Verona, Italy; ${ }^{12}$ Department of Oncology, AOU Città della Salute e della Scienza, Torino, Italy; ${ }^{13}$ Department of Molecular Biotechnology and Health Sciences, University of Torino, Torino, Italy; ${ }^{14} \mathrm{Vita}$-Salute San Raffaele University, Milano, Italy; ${ }^{15}$ German Cancer Consortium, Heidelberg, Germany; ${ }^{16}$ Unit of Immunogenetics, Leukemia Genomics and Immunobiology, IRCCS San Raffaele Scientific Institute, Milano, Italy and "Institute of Hematology "L. and A. Seràgnoli", University Hospital S.Orsola-Malpighi, Bologna, Italy

Acknowledgments: the authors would like to thank all investigators and data managers for their dedicated patient care.
Funding: this work was supported by the Italian Ministry of Health (RF-2011-02351998 to FC and LV, RF-2011-02348034 to LV, NS and AR, and TRANSCAN HLALOSS to LV and KF), by the Associazione Italiana per la Ricerca sul Cancro (Start-Up Grant \#14162 to LV), by the ASCO Conquer Cancer Foundation (2014 Young Investigator Award to LV), the Deutsche José Carreras Leukämie Stiftung (grants DJCLS R 15-02 and DJCLS 01 R/2017 to KF), the Josef Senker Stiftung to KF, and by the DKMS Mechtild Harf Foundation (DKMS Mechtild Harf Research Grant 2015 to LV and DKMS Mechtild Harf Research Grant 2018 to PC).

Correspondence: KATHARINA FLEISCHAUER

katharina.fleischhauer@uk-essen.de doi:10.3324/haematol.2019.225177

Information on authorship, contributions, and financial \& other disclosures was provided by the authors and is available with the online version of this article at www. haematologica.org.

\section{References}

1. Lee SJ, Klein J, Haagenson M, et al. High-resolution donor-recipient HLA matching contributes to the success of unrelated donor marrow transplantation. Blood. 2007;110(13):4576-4583.

2. Zino E, Frumento G, Marktel S, et al. A T-cell epitope encoded by a subset of HLA-DPB1 alleles determines nonpermissive mismatches for hematologic stem cell transplantation. Blood. 2004;103(4):14171424.

3. Fleischhauer K, Shaw BE, Gooley T, et al. Effect of T-cell-epitope matching at HLA-DPB1 in recipients of unrelated-donor haemopoietic-cell transplantation: a retrospective study. Lancet Oncol. 2012; 13(4):366-374.

4. Crocchiolo R, Zino E, Vago L, et al. Nonpermissive HLA-DPB1 disparity is a significant independent risk factor for mortality after unrelated hematopoietic stem cell transplantation. Blood. 2009; 114(7):1437-1444.

5. Pidala J, Lee SJ, Ahn KW, et al. Nonpermissive HLA-DPB1 mismatch increases mortality after myeloablative unrelated allogeneic hematopoietic cell transplantation. Blood. 2014;124(16):2596-2606.

6. Crivello P, Heinold A, Rebmann V, et al. Functional distance between recipient and donor HLA-DPB1 determines nonpermissive mismatches in unrelated HCT. Blood. 2016;128(1):120-129.

7. Arrieta-Bolaños E, Crivello $\mathrm{P}$, Shaw BE, et al. In silico prediction of nonpermissive HLA-DPB1 mismatches in unrelated HCT by functional distance. Blood Adv. 2018;2(14):1773-1783.

8. Petersdorf EW, Malkki M, O'hUigin C, et al. High HLA-DP Expression and Graft-versus-Host Disease. N Engl J Med. 2015; 373(7):599-609

9. Morishima S, Shiina T, Suzuki S, et al. Evolutionary basis of HLADPB1 alleles affects acute GVHD in unrelated donor stem cell transplantation. Blood. 2018;131(7):808-817.

10. Shaw BE, Gooley TA, Malkki M, et al. The importance of HLA-DPB1 in unrelated donor hematopoietic cell transplantation. Blood. 2007; 110(13):4560-4566.

11. Gagne K, Loiseau P, Dubois V, et al. Is there any impact of HLA-DPB1 disparity in 10/10 HLA-matched unrelated hematopoietic SCT? Results of a French multicentric retrospective study. Bone Marrow Transplant. 2015;50(2):232-236.

12. Dai S, Murphy GA, Crawford F, at al. Crystal structure of HLA-DP2 and implications for chronic beryllium disease. Proc Natl Acad Sci U S A. 2010;107(16):7425-7430.

13. Arrieta-Bolaños E, Crivello P, Metzing M, et al. Alloreactive T cell receptor diversity against structurally similar or dissimilar HLA-DP antigens assessed by deep sequencing. Front Immunol. 2018;9:280.

14. Gratwohl A. The EBMT risk score. Bone Marrow Transplant. 2012:47(6):749-756

15. Sorror ML, Maris MB, Storb R, et al. Hematopoietic cell transplantation (HCT)-specific comorbidity index: a new tool for risk assessment before allogeneic HCT. Blood. 2005;106(8):2912-2919. 